\title{
Effects of Late Surfactant Treatment Delivered After a Recruitment Maneuver on Respiratory Outcomes in Extremely Low Gestational Age Newborns: A Randomized Controlled Pilot Trial - LATE-REC-SURF Trial.
}

Alessandra Lio ( $\nabla$ alessandra.lio@policlinicogemelli.it)

Fondazione Policlinico Universitario Agostino Gemelli IRCCS https://orcid.org/0000-0001-6713-2719

Chiara Tirone

Fondazione Policlinico Universitario Agostino Gemelli IRCCS

Milena Tana

Fondazione Policlinico Universitario Agostino Gemelli IRCCS

Claudia Aurilia

Fondazione Policlinico Universitario Agostino Gemelli IRCCS

Rita Blandino

Fondazione Policlinico Universitario Agostino Gemelli IRCCS

Davide De Tomaso

Fondazione Policlinico Universitario Agostino Gemelli IRCCS

Francesca Fusco

Fondazione Policlinico Universitario Agostino Gemelli IRCCS

Angela Paladini

Fondazione Policlinico Universitario Agostino Gemelli IRCCS

Stefano Nobile

Fondazione Policlinico Universitario Agostino Gemelli IRCCS

Alessandro Perri

Fondazione Policlinico Universitario Agostino Gemelli IRCCS

Francesco Cota

Fondazione Policlinico Universitario Agostino Gemelli IRCCS

Roberta Pastorino

Fondazione Policlinico Universitario Agostino Gemelli IRCCS

Giovanni Vento

Università Cattolica del Sacro Cuore Facoltà di Medicina e Chirurgia: Universita Cattolica del Sacro Cuore Facolta di Medicina e Chirurgia 


\section{Study Protocol}

Keywords: Preterm newborns, Surfactant, bronchopulmonary dysplasia, chronic respiratory insufficiency of prematurity

Posted Date: January 3rd, 2022

DOI: https://doi.org/10.21203/rs.3.rs-1181889/v1

License: (c) (i) This work is licensed under a Creative Commons Attribution 4.0 International License.

Read Full License 


\section{Abstract \\ Background}

Mechanical ventilation is still needed in most preterm newborns, even in the non-invasive ventilation era. Ventilator-induced lung injury is one of the known pathogenetic factors of bronchopulmonary dysplasia (BPD) in preterm newborns. Lung injury has several patterns including surfactant dysfunction. Some recent trials have showed that a late surfactant administration can improve respiratory outcome in preterm babies still on invasive ventilation after the first week of life. Unfortunately, these results are still not conclusive. Moreover, giving surfactant after a recruitment manoeuvre in High Frequency Oscillatory Ventilation (HFOV) was shown to be safe and to reduce mortality in extremely preterm infants in a recent RCT. Our aim is to test the hypothesis that endotracheal administration of poractant-alfa preceded by a recruitment manoeuvre in HFOV in preterm infants still requiring mechanical ventilation at 7-10 days of life could facilitate extubation.

\section{Methods/Design:}

This will be an unblinded monocentric pilot trial that will be conducted in a III level Neonatal Intensive Care Unit at Fondazione Policlinico Agostino Gemelli IRCCS in Rome - Italy. Preterm newborns with a gestational age $<28$ weeks still requiring invasive mechanical ventilation at 7-10 days of life with a fraction of inspired oxygen $\left(\mathrm{FiO}_{2}\right)$ of more than 0.30 and/or an oxygenation index of 8 or more for at least 6 hours will be eligible for the study. Patients will be randomly assigned to intervention or to standard care. Intervention group infants will receive up to 4 doses of Poractant-alfa every 12 hours, each dose preceded by a recruitment manoeuvre in HFOV, until extubation. Primary endpoint will be the first successful extubation.

\section{Discussion}

Surfactant therapy is nowadays recommended in case of RDS in the first days of life but little is known about its effects in ventilator-dependant preterm newborns. Late administration of surfactant could help healing the lung of preterm babies in which RDS is evolving in a chronic pulmonary insufficiency of prematurity. The findings of this pilot trial will permit evaluation of the study design for a full-scale RCT.

\section{Trial registration:}

Clinicaltrials.gov - ID NCT04825197. Registered 12 April 2021, https://clinicaltrials.gov/ct2/home

\section{Background}


Despite the recent advances in perinatal care, bronchopulmonary dysplasia (BPD) still represents one of the most severe conditions in premature infants with longstanding consequences on pulmonary function and neurodevelopmental outcomes (1). BPD affects $22-38 \%$ of extremely low gestational age neonates (ELGANs; gestational age <28 weeks), which is the most vulnerable subset of preterm infants (2).

BPD is a complex condition that has a multifactorial pathogenesis. Key contributors include oxygen toxicity, ventilator-induced lung injury (VILI), inflammation and an arrest of lung vascular development. Mechanical ventilation (MV) is undoubtedly one of the key advances in neonatal care. Even in this era of noninvasive respiratory support, MV remains a mainstay of therapy in the extremely preterm population. Among survivors, almost $95 \%$ of preterm infants were invasively ventilated at some point during their hospital stay.

The patient determinants of VILI include preexisting lung injury, lung inflammation and surfactant abnormalities. Premature infants have lower lung tissue resilience due to immature collagen and elastin elements, surfactant and anti-oxidants in their lungs. Therefore, premature infants have a higher incidence of biophysical and biochemical lung injury when subjected to mechanical ventilation (3). In summary, VILI results in an inflammatory cascade that disrupts signaling pathways involved in lung development and repair and contributes to the development of BPD. The most severe mechanical consequences of acute lung injury occur when edematous material arising from the vasculature breaches the epithelial barrier and accumulates in the airspaces. The edematous material interferes with the functioning of pulmonary surfactant in the aerated regions. The resulting elevation in surface tension can dramatically reduce compliance and thus the pressure required to ventilate the lung (4). Thus, MV can interfere with surfactant metabolism and function leading to a worse respiratory condition and the need of aggressive ventilation which in turn increases lung injury. The application of mechanical ventilation alone alters surfactant and growth factor expression (5). Moreover, episodes of increased requirement for ventilatory support are associated with dysfunctional surfactant, which is primarily due to low surfactant protein B (SP-B) (6). Pulmonary surfactant has a complex metabolism which is slower in newborns (especially those born prematurely) than in adults or those with lung injury (7). Recently, two randomized controlled trials evaluated the effect of a late surfactant administration in preterm infants with severe respiratory distress that required prolonged invasive ventilation. They failed to demonstrate a reduction in the incidence of BPD at 36 weeks of postmenstrual age (PMA) among infants treated with late surfactant but showed some improvements in long-terms outcomes (less rehospitalization for pulmonary problems and less use of home respiratory support over the first year of life) (8-9). Moreover, in the study by Hascoet et al, the 2-year follow-up showed a statistically significant better growth among the treatment group patients compared to the control group ones. Nevertheless, both the studies were not designed to show an improvement in long-term outcomes. Indeed, the above-mentioned trials were inconclusive but encouraging, as they failed to demonstrate the objective they were designed for but still showed some good results in terms of long-term outcomes (respiratory morbidity at one year of age and growth at two years of age). Moreover, there are some substantial differences in the study design among the two trials that weaken the conclusion of both the trials and make new studies essential to unravel this matter. Recently, the IN-REC-SURE (INtubate, RECruit-SURfactant-Extubate) trial compared surfactant 
administration after alveoli recruitment using high frequency oscillatory ventilation (HFOV), followed by extubation with the traditional INSURE method in preterm infants who failed nasal CPAP. This RCT showed a decreased need of $\mathrm{MV}$ in babies who received INRECSURE compared to those who received INSURE method (10). In animal models, lung recruitment before surfactant administration improved gas exchange and lung function owing to a more homogeneous surfactant distribution (11-12). We hypothesized that optimizing end-expiratory lung volume before surfactant administration may improve the success rate of a late treatment with surfactant.

\section{Methods/design Study Design}

This protocol describes a pilot randomised, unblinded, controlled trial. The study flow is outlined in fig.1. The study will be held in a III level NICU in Fondazione Policlinico Agostino Gemelli - IRCCS in Rome Italy. The study will last 18 months and will involve 20 patients (10 for each group).

\section{Participants}

ELGANs still requiring invasive mechanical ventilation at 7-10 days of life with a fraction of inspired oxygen (FiO2) of more than 0.30 and/or an oxygenation index of 8 or more for at least 6 hours will be eligible for the study. Only infants for whom written consent from the parents or legal guardian will be obtained will be randomized. Infants will be randomly assigned to receive mechanical ventilation and poractant alfa (Treatment Group) or continue mechanical ventilation following local protocols (Control Group). Mechanical ventilation will be High Frequency Oscillatory ventilation in either group, since in our centre it is the elective modality for ELGANs. In the control arm, HFOV will be used according to the department protocol which includes the modification of the ventilatory parameters every 8-12 hours on the bases of the blood gases analyses, the SpO2 value and the chest x-rays. In both groups, extubation will be performed when a Mean Airway Pressure $<9 \mathrm{cmH} 2 \mathrm{O}$ and a FiO2 $<0.3$ will be required to maintain the SpO2 stably between 90 and $95 \%$ and the deltaP will not exceed $20 \mathrm{cmH}_{2} \mathrm{O}$ with a Frequency equal to $13-15 \mathrm{~Hz}$ to maintain pCO2 value between 45 and $55 \mathrm{mmHg}$ with. The patients enrolled will be 10 for each group. Enrolled infants will be under supervision in the NICU.

\section{Inclusion criteria}

1. Extremely low gestational age newborn infants (GA $<28$ weeks) - gestational age matching between maternal dates and/or early antenatal ultrasound

2. Singleton or multiple birth

3. Postnatal age between 7 and 10 days

4. Invasive mechanical ventilation still needed 
5. Fraction of inspired oxygen $\left(\mathrm{FiO}_{2}\right)$ of more than 0.30 and/or an oxygenation index of 8 or

6. more for at least 6 hours

7. Stable cardiovascular condition

8. Informed consent form signed by parents or legal guardian

\section{Exclusion Criteria}

1. Major congenital malformation (i.e., infants with genetic, metabolic or endocrine disorder diagnosed before enrolment)

2. High index of suspicion of infection before enrolment

3. Neurological conditions that might contraindicate extubation

4. Inotropic agents needed

5. Pneumothorax

6. Hemodynamically significant ductus arteriosus

7. Surgical intervention within the past 72 hours

8. Partecipation in another interventional clinical study that may interfere with the results of this trial

9. Known hypersensitivity to the drug or to one of the excipient

\section{Procedures}

Once the informed consent form is signed, eligibility will be confirmed. All inclusion and exclusion criteria must be confirmed within 24 hours prior to the first dose of study drug. Complete medical history and physical examination will be performed and blood tests will be documented from the infant's charts. Information regarding siblings and mother's medication during and after pregnancy will be collected. Screening procedures will be performed and recorded in the infant's source document and eCRF. Following screening procedures eligible infants will be randomly assigned to one of the two treatment groups. Randomization will be performed using random allocation generated by computer code. The randomization will be performed in permuted unequal blocks. The random allocation sequence will be generated using the module ralloc.ado in Stata/IC 16.1 (Stata-Corp, College Station, Texas). Concealment will be performed by closed envelopes.

After randomisation, during the treatment period, the following data will be recorded every day: physical examination, ventilation parameters, need of oxygen, $\mathrm{Ol}, \mathrm{pH}, \mathrm{pCO}_{2}, \mathrm{pO}_{2}, \mathrm{BE}$, vital signs as $\mathrm{SpO}_{2}$, Heart rate, Diastolic and Systolic Blood pressure, respiratory rate, Silverman Score; recording of concomitant medications; lung expansion at lung Xray if performed; weight; any adverse event. The same data will be recorded weekly until 36 weeks PMA and then at 40 weeks PMA or discharge whichever comes first.

Follow-up will continue after discharge until the first year of corrected term-age: babies will be visited at 3 months of corrected term-age and at one year of corrected term-age. During the follow-up visits, parents 
will fulfil a questionnaire regarding the respiratory condition of their baby at home. For all procedures see fig.2.

\section{Intervention}

Intervention group will receive up to 4 doses $(100 \mathrm{mg} / \mathrm{Kg})$ of Poractant alfa (Curosurf - Chiesi) every 12 hours; each dose will be preceded by a recruitment manoeuvre in HFOV. Optimal recruitment is defined as adequate oxygenation using a fraction of inspired oxygen $\left(\mathrm{FiO}_{2}\right)$ of 0.30 or less. The continuous distending pressure (CDP) will be increased stepwise $\left(1 \mathrm{cmH}_{2} \mathrm{O}\right.$ every $\left.2-3 \mathrm{~min}\right)$ as long as pulse oximetry $\left(\mathrm{SpO}_{2}\right)$ improves. The $\mathrm{FiO}_{2}$ will be reduced stepwise, keeping $\mathrm{SpO}_{2}$ within the target range (87-94\%). The recruitment procedure will be stopped if oxygenation no longer improves or if the $\mathrm{FiO}_{2}$ is equal to or less than 0.30 . The corresponding CDP will be called the opening pressure (CDPO). Next, the CDP will be reduced stepwise (1-2 $\mathrm{cmH}_{2} \mathrm{O}$ every 2-3 min) until the $\mathrm{SpO}_{2}$ deteriorates (by at least 2-3 points). The corresponding CDP will be called the closing pressure (CDPC). After a second recruitment maneuver at CDPO for $5 \mathrm{~min}$, the optimal CDP (CDPOPT) will be set $2 \mathrm{cmH}_{2} \mathrm{O}$ above the CDPC for at least $3 \mathrm{~min}$. All infants will receive caffeine at a dose of $5 \mathrm{mg} / \mathrm{kg} / \mathrm{die}$. All infants will receive remifentanil infusion during mechanical ventilation $(0.075-0.3 \mathrm{mcg} / \mathrm{kg} / \mathrm{min})$. Infants still requiring MV at days 13 of age will be started on the low dose corticosteroid regime used in the DART study by Doyle et al (13).

\section{Outcome measures}

Primary outcome: The time to first successful extubation (day of life).

Defining an extubation as "successful" is a very hard task, since in preterm newborns many reintubation occur for extra-respiratory reasons. In a recent study, reintubations due to non-respiratory causes were negligible in the first week after extubation, but became much more frequent after 14 days. For these reasons, we chose to define an extubation "successful" if it is not followed by a reintubation in the subsequent 7 days (14). This will provide a more complete overview of the true reintubation rates, making them easier to compare. Extubation criteria include: $\mathrm{FiO}_{2}$ less than 0.30 for arterial oxygen percent saturation between $88 \%$ and $92 \%$ and partial pressure of carbon dioxide between 40 and $55 \mathrm{~mm} \mathrm{Hg}$ for at least 6 hours with a MAP $\leq 9 \mathrm{cmH}_{2} \mathrm{O}$. Criteria for reintubation: 6 episodes of apnea and/or bradycardia that require stimulation over a 6-hour period; 1 episode of apnea and/or bradycardia that require resuscitation with positive pressure; $\mathrm{FiO}_{2}$ greater than 0.40 with a $\mathrm{MAP}>8 \mathrm{cmH}_{2} \mathrm{O}$ for more than 4 hours; or partial pressure of carbon dioxide greater than $60 \mathrm{mmHg}$ and a $\mathrm{pH}<7.20$; Silvermann Score $>6$; respiratory rate $>100 / \mathrm{min}$; haemodynamic instability; need of surgery; new onset of contraindications to non invasive ventilation. All babies will be on Caffeine therapy at a dosage of $5 \mathrm{mg} / \mathrm{kg} / \mathrm{die}$.

Secondary outcomes: incidence of pneumothorax, incidence of interstitial emphysema, incidence of pulmonary haemorrhage, incidence of necrotising enterocolitis, incidence of intraventricular haemorrhage 
occurring after the randomization, incidence of periventricular leukomalacia, and chronic lung disease of prematurity, length of hospital stay, length of mechanical ventilation, length

of oxygen-therapy, length of respiratory support, use of post-natal steroids. All surviving infants will be reviewed for follow-up at 1 year of age. Parents will be interview to record all events occurring after discharge (rehospitalisation for respiratory problems with the need for reintubation, invasive ventilation, and oxygen supplementation; corticosteroid use after discharge; outpatient visits for respiratory problem). Ventilator, clinical and blood gases data will be recorded during the procedure and every 12 hours in the first 3 days after intervention. A lung ultrasound will be performed before the first intervention and then daily until extubation or for the following 3 days.

\section{Statistical analysis}

No formal sample size calculation was performed because this is a pilot study whose results will permit to design a full-scale RCT. Revising our past records, we estimate that there will be a number of 20-25 patients having the eligibility criteria in the study period. Therefore, we chose to recruit 10 patients in each group. Clinical characteristics of infants will be described using mean values and standard deviation, median value and range, or rate and percentage. Univariate statistical analysis will be performed using the Student " $\mathrm{t}$ " test for parametric continuous variables, the Wilcoxon rank-sum test for non-parametric continuous variables, and Fisher's exact test for categorical variables. A $p<0.05$ will be considered statistically significant.

\section{Data management}

The Sponsor's designees will monitor all aspects of the study carefully with respect to ICH GCPs and SOPs for compliance with applicable government regulations. eCRF will be periodically monitored and source verified against corresponding source documentation (e.g., office and clinical laboratory records) for each subject. Clinical monitors will evaluate periodically the progress of the study, including the verification of appropriate consent form procedures, review of drug accountability and study drug preparation procedures, adherence to dosing procedures, the investigator's adherence to the protocol, maintenance of records and reports, review of source documents for accuracy, completeness, and legibility, and review of study regulatory documents, including, but not limited to: study agreement, study insurance. In addition, the monitor shall review completed eCRF and study documentation for accuracy and completeness, and protocol compliance. The monitor should assure that data captured in the eCRF is fully supported by the source documents.

\section{Discussion}

Despite the enormous advances in perinatal medicine, BPD still represents a burden in neonatology. Its incidence has not changed in the past decades, although a big effort has been made to identify 
preventive strategies (1). Children with a history of BPD have a significant risk of rehospitalization and death in the first years of life. Moreover, BPD have a big impact on pulmonary and cardiac function later in childhood. As well as cardiorespiratory worse outcomes, infants with BPD has worse neurologic outcomes including a lower head circumference, cerebral palsy, and lower cognitive and language skills (1). Therefore, prevention of BPD remains one of the most important goal in the NICUs all over the world.

Little evidence exists about beneficial effects of late surfactant in improving respiratory outcomes in preterm infants (8-9). Surely, available data are not conclusive but they are still encouraging. The possibility to use a well-known drug to prevent BPD is thrilling and our aim is to study in deep and optimize its late use.

This pilot study will provide essential information about the safety profile of poractant-alpha in preterm infants after the first week of life as well as preliminary data about its efficacy in decreasing MV when combined with a recruitment manoeuvre. If this strategy will prove as safe and beneficial in a small population of ELGANs, a full-scale multicentre RCT will be designed.

\section{Abbreviations}

NICU Neonatal Intensive Care Unit

BPD Bronchopulmonary Dysplasia

ELGANs Extremely low gestational age newborns

MV Mechanical Ventilation

VILI Ventilation Induced Lung Injury

RDS Respiratory Distress Syndrome

HFOV High Frequency Oscillatory Ventilation

GA Gestational Age

BW Birth Weight

PMA Post-mestrual age

MAP Mean Airway Pressure

CDP Continuous Distending Pressure

Ol Oxygenation Index

\section{Declarations}


Ethics approval and consent to participate

Ethical approval for this study was obtained through Università Cattolica del Sacro Cuore Ethics Committees for study protocol version 2 dated 16/12/2021 (protocol number 3435) and all supporting documents.

\section{Competing interests}

The authors declare that they have no competing interests.

$\underline{\text { Consent for publication }}$

Not applicable

\section{Funding}

Chiesi Farmaceutics will give a liberal contribution not exceeding the $30 \%$ of all the cost of the study. Internal funds will be used to cover the remaining costs.

\section{Authors' contributions}

$\mathrm{AL}$ gave substantial contributions to the conception and design of the research and drafted the article. CT contributed to the design of the research and revised the paper critically for important intellectual content. MT revised the paper critically for important intellectual content. CA revised the paper critically for important intellectual content. RB collected and interpreted data to optimize statistical design and revised the paper critically for important intellectual content. DD collected and interpreted data to optimize statistical design and revised the paper critically for important intellectual content. FF revised the paper critically for important intellectual content. APa revised the paper critically for important intellectual content. SN revised the paper critically for important intellectual content. APe revised the paper critically for important intellectual content. FC gave substantial contribution to the statistical design of the work and revised the paper critically for important intellectual content. RP gave substantial contribution to the statistical design of the work and revised the paper critically for important intellectual content. GV gave substantial contributions to the conception and design of the work and revised the paper critically for important intellectual content. All the authors approve the final version to be published, agree to be accountable for all aspects of the work and ensure that questions related to the accuracy or integrity of any part of the work will be appropriately investigated and resolved.

Data and material availability.

Data will be available on request from the authors.

\section{Acknowledgements}


We thank Chiesi Farmaceutici (Parma, Italy) for partially funding this trial. Their generosity will permit payment of a Contract Research Organization that will help us in monitoring and drug surveillance activities.

\section{References}

1. Hwang JS, Rehan VK. Recent Advances in Bronchopulmonary Dysplasia: Pathophysiology, Prevention, and Treatment. Lung. 2018 Apr;196(2):129-38. doi:10.1007/s00408-018-0084-z.

2. Poets CF, Lorenz L. Prevention of bronchopulmonary dysplasia in extremely low gestational age neonates: current evidence. Arch Dis Child Fetal Neonatal Ed. 2018;0:F1-7.

Doi:10.1136/archdischild-2017-314264.

3. Kalikkot Thekkeveedu R, Guaman MC, Shivanna B. Bronchopulmonary dysplasia: A Review of Pathogenesis and Pathophysiology. Respir Med. 2017 November;132:170-7. doi:10.1016/j.rmed.2017.10.014.

4. Bates JH, Smith BJ. Ventilator-induced lung injury and lung mechanics. Lung. 2018 April;196(2):129-38. doi:10.21037/atm.2018.06.29.

5. Digeronimo RJ, Mustafa SB, Ryan RM, Sternberg ZZ, Ashton DJ. Seidner SR Mechanical ventilation down-regulates surfactant protein $A$ and keratinocyte growth factor expression in premature rabbits. Pediatr Res. 2007;62(3):277-82. doi:10.1203/PDR.0b013e3181256aeb.

6. Merrill JD, Ballard RA, Cnaan A, Hibbs AM, Godinez RI, Godinez MH, et al. Dysfunction of pulmonary surfactant in chronically ventilated premature infants. Pediatr Res. 2004;56:1-9. doi:10.1203/01.PDR.0000145565.45490.D9.

7. Nkadi PO, Merritt TA, Pillers DA. An Overview of Pulmonary Surfactant in the Neonate: Genetics, Metabolism, and the Role of Surfactant in Health and Disease. Mol Genet Metab. 2009 June; 97(2) 95-101. doi: 10.1016/j.ymgme.2009.01.015.

8. Hascoët JM, Picaud JC, Ligi I, Blanc T, Moreau F, Pinturier MF, et al. Late Surfactant Administration in Very Preterm Neonates With Prolonged Respiratory Distress and Pulmonary Outcome at 1 Year of Age: A Randomized Clinical Trial. JAMA Pediatr. 2016 Apr;170(4):365-72. doi:10.1001/jamapediatrics.2015.4617.

9. Ballard RA, Keller RL, Black DM, Ballard PL, Merrill JD, Eichenwald EC, et al. Randomized Trial of Late Surfactant Treatment in Ventilated Preterm Infants Receiving Inhaled Nitric Oxide. J Pediatr. 2016 Jan;168:23-9.

.e4

. doi: 10.1016/j.jpeds.2015.09.031.

10. Vento G, Ventura ML, Pastorino R, van Kaam AH, Carnielli V, Cools F, et al. Lung recruitment before surfactant administration in extremely preterm neonates with respiratory distress syndrome (IN-RECSUR-E): a randomised, unblinded, controlled trial. Lancet Respir Med. 2021 Feb;9(2):159-66. doi:10.1016/S2213-2600(20)30179-X. 
11. Krause MF, Jäkel C, Haberstroh J, Schulte-Mönting J, Leititis JU, Orlowska-Volk M. Alveolar recruitment promotes homogenous surfactant distribution in a piglet model of lung injury. Pediatr Res. 2001;50:34-43. doi:10.1203/00006450-200107000-00009.

12. Tingay DG, Togo A, Pereira-Fantini PM, Miedema M, McCall KE, Perkins EJ, et al. Aeration strategy at birth influences the physiological response to surfactant in preterm lambs. Arch Dis Child Fetal Neonatal Ed. 2019 Feb 1. pii: fetalneonatal-2018-316240. doi:10.1136/archdischild-2018-316240.

13. Doyle LW, Davis PG, Morley CJ, McPhee A, Carlin JB. Low-dose dexamethasone facilitates extubation among chronically ventilator-dependent infants: a multicenter, international, randomized, controlled trial. Pediatrics. 2006;117:75-83. doi:10.1542/peds.2004-2843.

14. Shalish W, Latremouille S, Papenburg J, Sant'Anna GM. Patterns of reintubation in extremely preterm infants: a longitudinal cohort study. JAMA Pediatr. 2019 Dec 20. doi:10.1001/jamapediatrics.2019.4868.

\section{Figures} Participants are identified in the NICU of Fondazione Policlinico Agostino Gemelli- IRCCS.
Eligible patients are ELGANs still requiring invasive mechanical ventilation at 7-10 days of life with a fraction of inspired oxygen (FiO2) of more than 0.30 and/or
an oxygenation index of 8 or more for at least 6 hours.

Parents or legal guardians of the eligible patient is approached and provided with information sheet. If they are willing to let their child participate to the trial they are asked to firm informed consent.

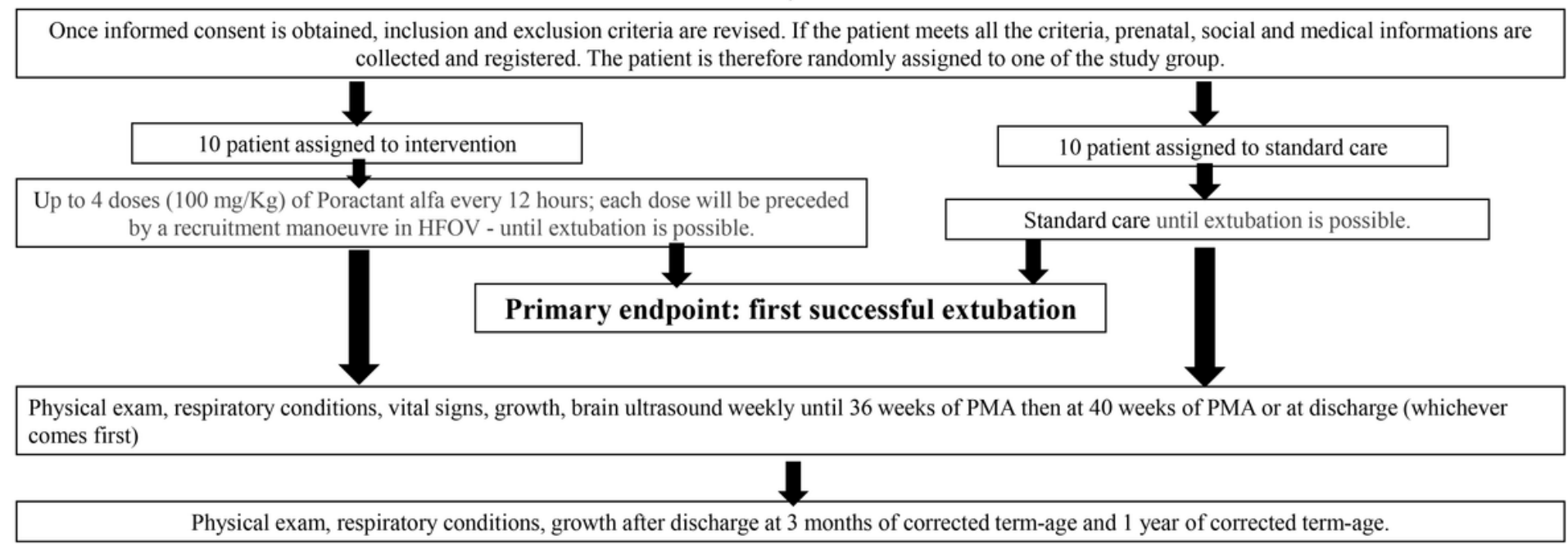

\section{Figure 1}

This protocol describes a pilot randomised, unblinded, controlled trial 


\begin{tabular}{|c|c|c|c|c|c|c|c|}
\hline & $\begin{array}{l}\text { Screening } \\
\text { (DOL 8- } \\
10)^{1}\end{array}$ & Randomisation & $\begin{array}{l}\text { Treatment } \\
\text { Period } \\
(\text { Day } 1-3)^{2}\end{array}$ & $\begin{array}{l}\text { Treatment- } \\
\text { to-discharge } \\
\text { period } \\
\text { Weekly }\end{array}$ & $\begin{array}{l}\text { Post- } \\
\text { discharge } \\
\text { Term- } \\
\text { equivalent } \\
\text { age }( \pm 14 \\
\text { days) }\end{array}$ & $\begin{array}{l}\text { Post- } \\
\text { discharge } \\
3 \text { months } \\
\text { corrected } \\
\text { age } \\
( \pm 14 \text { days })\end{array}$ & $\begin{array}{l}\text { Post- } \\
\text { discharge } \\
12 \text { months } \\
\text { corrected } \\
\text { age }( \pm 14 \\
\text { days) } \\
\text { Final study }\end{array}$ \\
\hline Informed consent & + & & & & & & \\
\hline $\begin{array}{l}\text { Inclusion/exclusion } \\
\text { criteria }\end{array}$ & + & + & & & & & \\
\hline Demographics & + & & & & & & \\
\hline $\begin{array}{l}\text { Maternal/Parental } \\
\text { data }\end{array}$ & + & & & & & & \\
\hline Physical exam & + & & + & + & + & + & + \\
\hline Vital signs & + & & + & + & + & + & + \\
\hline Body Weight & + (birth) & & + & + & + & + & + \\
\hline Medical History & + & & & & & & \\
\hline $\begin{array}{l}\text { Medication taken } \\
\text { since birth }\end{array}$ & + & & & & & & \\
\hline Head circumference & + (birth) & & + & + & + & + & + \\
\hline Body Length & + (birth) & & + & + & + & + & + \\
\hline Treatment procedure & & & + & & & & \\
\hline $\begin{array}{l}\text { Ventilation } \\
\text { parameters }\end{array}$ & + & & + & + & + & + & + \\
\hline $\mathrm{pH}, \mathrm{pCO} 2, \mathrm{pO} 2, \mathrm{BE}$ & + & & + & + & & & \\
\hline Need of oxygen, OI & + & & + & + & + & + & + \\
\hline $\begin{array}{l}\text { Oral food } \\
\text { consumption }\end{array}$ & + & & + & + & + & + & + \\
\hline Adverse event & & & + & + & + & + & + \\
\hline $\begin{array}{l}\text { Concomitant } \\
\text { medication }\end{array}$ & & & + & + & + & + & + \\
\hline $\begin{array}{l}\text { Parents questionnaire } \\
\text { on respiratory } \\
\text { condition after } \\
\text { discharge }\end{array}$ & & & & & & & + \\
\hline $\begin{array}{l}\text { Lung expansion at } \\
\text { Xray }\end{array}$ & & & + & & & & \\
\hline Brain Ultrasound & + & & & + & & & \\
\hline
\end{tabular}

${ }^{1}$ DOL day of life

${ }^{2}$ Twice daily

\section{Figure 2}

\section{Study procedures}

